\title{
La crisis del sistema de partidos políticos en la Argentina
}

\section{The crisis of the system of political parties in Argentina}

\author{
Leandro López
}

\begin{abstract}
Resumen
Es importante analizar la problemática democrática en función del comportamiento de los partidos políticos. En este sentido, centrar el estudio de la democracia en torno al sistema de partidos, lejos de ser un mero análisis electoral, supone trabajar y teorizar en relación a los elementos esenciales que hacen a la calidad institucional y a la necesaria gobernabilidad que supone toda democracia. Partiré de las teorizaciones básicas existentes para abordar la problemática del sistema de partidos, para así llegar a analizar el caso concreto de la democracia en la Argentina actual, donde el problema de la reconfiguración o recomposición partidaria se hace sumamente imperioso para el fortalecimiento del sistema.

La necesidad de una imperiosa reconstrucción de un sistema de partidos sólidos en la Argentina es una prioridad de nuestra democracia. Para analizar el modelo de incertidumbre actual donde vivimos una democracia de candidatos o personalidades y no de partidos, propongo analizar el tema no solo desde los datos actuales sino remontándome al origen de la problemática, la cual tiene un arrastre y una significación histórica y política que me parece importante traer a colación, ya que contribuye en términos de aportes a fortalecer nuestros sistemas democráticos.

Palabras clave: sistema de partidos políticos, sistema democrático, representación política, personalización de la política, institucionalización.

\section{Abstract}

It is important to analyze the role of the democracy problematic based on the behavior of the political parties. In this sense, focusing the study of democracy around the system matches, far from being a mere electoral analysis, means working and theorizing on the essential elements that create institutional quality and the necessary governance involved in any democracy. I operate from the basic theorizations existing to deal with the problems of the party system to analyze the case of the current Argentina
\end{abstract}


democracy, where the problem of the reconfiguration or recomposition of parties becomes extremely imperative for strengthening the system.

The compelling need of a reconstruction of a solid system of parties in Argentina is a priority of our democracy. To analyze the model of current uncertainty where we live a democracy of candidates or personalities, not parties, I propose to analyze the issue not only from the current data but going back to the origin of the problem, which bears a political and historical meaning, which seems important for me to bring up, since it can help to contribute to strengthen our democratic systems.

Keywords: system of political parties, democratic system, political representation, personalization of politics, institutionalization. 


\section{Introducción}

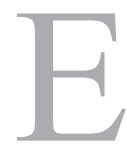

s importante analizar la problemática democrática en función del comportamiento de los partidos políticos. En este sentido, centrar el estudio de la democracia en torno al sistema de partidos lejos de ser un mero análisis electoral, supone trabajar y teorizar en relación a los elementos esenciales que hacen a la calidad institucional y a la necesaria gobernabilidad que supone toda democracia. En este sentido podemos considerar tomando a Bobbio que "la democracia, como método, está abierta a todos los posibles contenidos, pero a la vez es muy exigente en el pedir respeto para las instituciones, porque precisamente en esto reposan todas las ventajas del método; entre estas instituciones están los partidos, únicos sujetos autorizados para fungir como mediadores entre los individuos y el gobierno" (1984: 18-19).

Es indudable la relación existente entre sistema democrático y partidos políticos, los cuales son reconocidos en el caso particular de Argentina por el derecho constitucional mediante el artículo 38 de la Constitución Nacional que los reconoce como "instituciones fundamentales para el sistema democrático". Siguiendo en esta línea de argumentación, podemos agregar según Dahlque "probablemente ninguna institución política conforma tanto el paisaje político de un país democrático como su sistema electoral y de partidos políticos. Y ninguna despliega tanta variedad" (1998: 151).

En relación a ello, partiré de las teorizaciones básicas existentes para abordar la problemática del sistema de partidos para llegar a analizar el caso concreto de la democracia en la Argentina actual, donde el problema de la reconfiguración o recomposición partidaria se hace sumamente imperioso para el fortalecimiento del sistema. 
La necesidad de una imperiosa reconstrucción de un sistema de partidos sólidos en la Argentina es una prioridad de nuestra democracia. Para analizar el modelo de incertidumbre actual donde vivimos una democracia de candidatos o personalidades y no de partidos, propongo analizar el tema no solo desde los datos actuales sino remontándome al origen de la problemática, la cual tiene un arrastre y una significación histórica y política que me parece importante traer a colación, ya que contribuye en términos de aportes a fortalecer nuestros sistemas democráticos.

\section{El sistema de partidos tradicional y su implosión}

Propongo como punto inicial de discusión tomar la definición que hace Alan Ware de partidos políticos: "un partido político es una institución que (a) busca influencia en el seno de un Estado, a menudo intentando ocupar posiciones en el gobierno, y (b) puesto que normalmente defiende más de un único interés social intenta, hasta cierto punto, agregar intereses" (1996: 32).

Esta definición de Ware en primer lugar hace referencia al papel central que ocupa el Estado como ámbito de actuación de los partidos; en segundo lugar, reconoce que para muchos partidos la búsqueda del poder es ocupar el gobierno; en tercer lugar, distingue entre partidos políticos y grupos de presión; finalmente, excede el marco ideológico para analizar los mismos.

En relación a la definición de sistema de partidos políticos, creo que la propuesta de Dieter Nohlen representa acabadamente lo que interpreto de este: "Por sistema de partido se entiende la composición estructural de la totalidad de los partidos políticos en un estado. Los elementos o interrogantes mencionados, tratados por los investigadores de los sistemas de partidos son los siguientes: a) el número de partidos, b) su tamaño, c) la distancia ideológica entre ellos, d) sus pautas de interacción, e) su relación con la sociedad o grupos sociales, y f) su actitud frente al sistema político." (1994: 41). 
Centrándonos en los aspectos epistemológicos que dan cuenta de los partidos políticos en la modernidad, vemos que su origen está ligado a la construcción y consolidación del Estado-nación. Para dar cuenta de ello, existen dos tipos principales de explicaciones para el nacimiento de los partidos que si bien se distinguen, son complementarios.

En primer lugar, el Enfoque Institucional nace relacionado con el desarrollo de la institución característica de la democracia moderna, el parlamento. Su principal referente, Maurice Duverger (1974), distingue entre partidos de creación interna que son aquellos que nacen en el mismo seno del parlamento nucleados bajo diferentes objetivos que se van vertebrando e institucionalizando; y partidos de creación externa que nacen a fines del siglo XIX y comienzos del siglo XX, a partir de la lucha por la extensión del sufragio. Es así como aparecen los comités electorales, que surgen por un impulso central y bajo la influencia de grupos exógenos que realizan todas sus actividades por fuera del parlamento.

En segundo lugar, el Enfoque Sociológico o Genético remite a ciertas divisiones sociales estructurales, acaecidas durante los procesos de formación de los Estados y de la economía moderna. Poniendo su eje en las transformaciones económicas y desde allí la construcción y consolidación de las alternativas partidarias.

En el caso concreto de la Argentina, y de ahí que aclarara que ambos enfoques a pesar de su distinción están ligados, vemos que el desarrollo partidario se dio en forma pausada y bajo la combinación de estos dos enfoques.

El primer partido político moderno de la Argentina fue la Unión Cívica Radical (UCR), si tomamos los enfoques mencionados párrafos arriba podemos concluir que resultó de la combinación de elementos institucionales con creación externa, debido a la necesidad de establecer normas de 
procedimiento electoral claves que terminan con la sanción de la primera ley electoral argentina moderna en 1912 conocida como la "Ley Sáenz Peña" que estableció la universalidad del voto masculino, secreto, obligatorio y que finalmente creó las condiciones para que llegue al poder en 1917 Hipólito Yrigoyen como el primer presidente electo democráticamente en la Argentina.

Desde ya que sería imposible negar los elementos sociológicos de una sociedad emergente donde los hijos y nietos de los primeros inmigrantes querían y necesitaban participar en la vida institucional del país. Esta irrupción del radicalismo no se vio acompañada por el surgimiento de otro partido político moderno, sino que convivió con el viejo partido conservador, cuya construcción era la suma de múltiples acuerdos de grupos de élite pero sin ideología ni estructuración alguna. Esta razón hace que la democracia argentina en 1916 sea un problema en términos de competitividad y construcción de un sistema de partidos políticos modernos. De allí que la sucesión presidencial de 1922 sea dentro de las filas del partido radical donde sí se pueden ver la construcción de dos líneas internas claramente diferenciadoras pero aglutinadas bajo un signo partidario.

Finalmente el retorno al poder de Yrigoyen al gobierno en 1928 marcó la supremacía de la UCR en la competencia electoral e inauguró a partir de 1930 una larga y lamentable práctica de recurrir a los golpes militares para resolver los problemas que se deberían resolver en lo electoral.

Es recién en 1945 donde surge el otro partido político moderno de la Argentina, aunque con características movimientistas que es el peronismo. Al igual que el radicalismo, se da por una combinación de elementos institucionales que permitieron la creación externa del partido y principalmente el elemento sociológico que le da su fuerte componente movimientista, el peronismo esta intrínsecamente ligado a la figura de Juan Domingo Perón y a las reivindicaciones obreras que supo encarnar, producto de un incipiente proceso de industrialización y urbanización en las urbes de 
las grandes ciudades. Hijos y nietos de inmigrantes que huían de la guerra y que demandaban la construcción de un estado benefactor que permitiera la movilidad social ascendente, de allí su histórica vinculación con los sindicatos y organizaciones obreras. Vale aclarar que en este periodo se logra la ampliación del voto a la mujer, quienes por primera vez pueden ejercer los derechos civiles y políticos en la elección de 1952 donde Perón es reelecto por amplio margen. Sin embargo, la tendencia hegemónica del peronismo hizo imposible un modelo de alternancia y competitivo, razón por la cual, la etapa del peronismo 1945-1955 fue más un modelo plebiscitario o cesarista que un modelo democrático pleno.

La etapa del peronismo en el poder terminó con el golpe de 1955 y al igual que al radicalismo en 1930, le sucedió una etapa de prohibiciones hasta el año 1973 donde recién ahí fue vuelto a legalizar el partido y se permitió la participación electoral.

Estas dos etapas históricas están signadas por el surgimiento de los dos principales partidos políticos que van a signar la historia política argentina, aunque es importante mencionar las interrupciones constitucionales constantes al desarrollo democrático, las prohibiciones cuando se dieron aperturas semidemocráticas, hicieron que no se viva en un estado pleno de democracia hasta el año 1983 donde ahí Argentina deja definitivamente atrás las experiencias autoritarias de los gobiernos cívicos militares y comienza a vivir un sistema competitivo de alternancia en el poder.

El inicio de redemocratización en Argentina inauguró por primera vez en nuestra historia reciente la competencia igualitaria entre los dos viejos partidos políticos nacidos tanto por reivindicaciones políticas de la UCR como por reivindicaciones sociales del peronismo. Tal es así que en 1983 el peronismo fue derrotado por primera vez en su historia y pasó a ocupar un rol absolutamente nuevo que era ser oposición dentro de un marco democrático. 


\section{Tabla 1.}

Elección presidencial 1983

\begin{tabular}{|c|c|c|c|c|}
\hline Fórmula & Partido & Votos & Porcentaje & Electores \\
\hline $\begin{array}{l}\text { Raúl Alfonsín - } \\
\text { Víctor Martínez }\end{array}$ & $\begin{array}{l}\text { Unión Cívica } \\
\text { Radical }\end{array}$ & 7724559 & 51,75 & 317 \\
\hline $\begin{array}{l}\text { Ítalo Luder - } \\
\text { Deolindo Felipe } \\
\text { Bittel }\end{array}$ & $\begin{array}{l}\text { Partido Justi- } \\
\text { cialista }\end{array}$ & 5995402 & 40,16 & 259 \\
\hline $\begin{array}{l}\text { Oscar Alende - } \\
\text { Lisandro Viale }\end{array}$ & $\begin{array}{l}\text { Partido Intran- } \\
\text { sigente }\end{array}$ & 347654 & 2,33 & 2 \\
\hline $\begin{array}{l}\text { Rogelio Frigerio } \\
\text { - Antonio Salona }\end{array}$ & $\begin{array}{l}\text { Movimiento de } \\
\text { Integración } \mathrm{y} \\
\text { Desarrollo }\end{array}$ & 177426 & 1,19 & 2 \\
\hline Otros & & 541751 & 3,63 & 20 \\
\hline Votos válidos & & 14927512 & 97,25 & \\
\hline Votos en blanco & & 334946 & 2,18 & \\
\hline Votos anulados & & 87728 & 0,57 & \\
\hline Votos totales & & 15350186 & 100 & 600 \\
\hline
\end{tabular}

Fuente: Dirección Nacional Electoral Argentina DINE Elaborado por: Autor.

\section{Del bipartidismo moderado al multipartidismo}

Utilizando un enfoque morfológico para analizar el sistema de partidos, y siguiendo con Duverger quien clasifica los partidos según el número de unidades que compiten en él, podemos decir que Argentina vivió un bipartidismo no del estilo norteamericano, pero si moderado. Según este autor el bipartidismo presenta las siguientes ventajas:

- Ordena de modo claro el sistema político oponiendo dos interlocutores fácilmente diferenciables. 
- Modera a los partidos ya que existen posibilidades de alternancia.

- Ofrece a los votantes la posibilidad de premiar o castigar al partido mediante el voto.

La principal crítica a este sistema es que sólo puede lograrse al costo de excluir partidos minoritarios de la posibilidad de contar con la representación parlamentaria. Sin embargo, esta posibilidad no estaba dada en la Argentina, porque hasta 1999 siempre existió un tercer partido que garantizó representación, sin contar un innumerable número de partidos con representación en cada una de las provincias que obtenía importantes números de escaños.

Este sistema bipartidista se reafirma en las elecciones de 1989 donde vuelve a ganar el peronismo la elección presidencial y provoca un doble acontecimiento histórico: en primer lugar, que un presidente coloque la banda presidencial, algo que no sucedía desde 1928 cuando Alvear le traspasa el mando a Yrigoyen; y en segundo lugar, que un presidente constitucional le traspase el poder a otro presidente constitucional de otro partido político, algo que no ocurría desde 1916 con la primer elección de Yrigoyen. 


\section{Tabla 2.}

Elección presidencial 1989

\begin{tabular}{|c|c|c|c|c|}
\hline Fórmula & Partido & Votos & Porcentaje & Electores \\
\hline $\begin{array}{l}\text { Carlos } \\
\text { Menem - } \\
\text { Eduardo } \\
\text { Duhalde }\end{array}$ & $\begin{array}{l}\text { Frente Justicia } \\
\text { Popular PJ-PI- } \\
\text { PCRA-PDC-PSA }\end{array}$ & 7953301 & 47,49 & 312 \\
\hline $\begin{array}{l}\text { Eduardo } \\
\text { Angeloz - } \\
\text { Juan Manu- } \\
\text { el Casella }\end{array}$ & $\begin{array}{l}\text { Unión Cívica } \\
\text { Radical }\end{array}$ & 5433369 & 32,45 & 231 \\
\hline $\begin{array}{l}\text { Álvaro } \\
\text { Alsogaray } \\
\text { - Alberto } \\
\text { Natale }\end{array}$ & $\begin{array}{l}\text { Alianza de Cen- } \\
\text { tro UCeDé-PDP }\end{array}$ & 1150603 & 6,87 & 33 \\
\hline $\begin{array}{l}\text { Eduardo } \\
\text { Angeloz } \\
\text { - María } \\
\text { Cristina } \\
\text { Guzmán }\end{array}$ & $\begin{array}{l}\text { Confederación } \\
\text { Federalista Inde- } \\
\text { pendiente }\end{array}$ & 728125 & 4,59 & 21 \\
\hline $\begin{array}{l}\text { Néstor Vi- } \\
\text { cente - Luis } \\
\text { Zamora }\end{array}$ & $\begin{array}{l}\text { Izquierda Unida } \\
\text { PCA-MAS }\end{array}$ & 409751 & 2,45 & 1 \\
\hline $\begin{array}{l}\text { José Corzo } \\
\text { Gómez - } \\
\text { Federico } \\
\text { Houssay }\end{array}$ & $\begin{array}{l}\text { Blanco de los } \\
\text { Jubilados }\end{array}$ & 317934 & 1,9 & 7 \\
\hline \multicolumn{2}{|c|}{ Partidos de distritos locales } & 316492 & 1,89 & 13 \\
\hline \multicolumn{2}{|c|}{ Votos positivos } & 16746257 & 98,01 & \\
\hline \multicolumn{2}{|c|}{ Votos en blanco } & 221585 & 1,3 & \\
\hline \multicolumn{2}{|c|}{ Votos anulados } & 115686 & 0,68 & \\
\hline
\end{tabular}

Fuente: Dirección Nacional Electoral Argentina DINE Elaborado por: Autor. 
Las primeras imágenes de la crisis del sistema de partidos en Argentina que llevan o conducen al fin del bipartidismo y el tránsito al multipartidismo se dan por algunos motivos que vale la pena resaltar:

1. La llegada de Menem al poder y su alianza con sectores conservadores que históricamente combatían al peronismo hizo que grupos progresistas se apartaran del movimiento y formaran un nuevo partido político, llamado Frente Grande (FG) que posteriormente va a tener un rol central en nuestra historia política.

2. La hiperinflación y la salida anticipada del poder por parte de Alfonsín, hicieron que la UCR viva un periodo de debilidad extrema al punto de dejar de ser competitiva a nivel nacional, aunque conservando poder en algunas provincias y municipios del interior.

3. La ley de convertibilidad, estableciendo que un peso argentino corresponde a un dólar norteamericano, permitió rápidamente al gobierno controlar la inflación y pasar a un esquema de estabilidad (aunque con un costo futuro terrible y consecuencias nefastas una década después). Este hecho convirtió a Menem en el personaje rutilante de la época, lo cual volvió a mostrar la tendencia hegemónica que había tenido históricamente el peronismo.

En este sentido, tanto las primeras elecciones legislativas nacionales (1991) con Menem en el poder como las siguientes (1993) marcan en primer lugar la amplia supremacía electoral del peronismo, en segundo lugar, la caída y el deterioro de la UCR como fuerza con capacidad para ganar una elección, y en tercer lugar, la irrupción del FG como alternativa creciente sobre todo en los grandes centros urbanos. 
Esta situación de superioridad electoral y de liderazgo de Menem llevó la discusión a consolidar el intento hegemónico que suponía la reelección presidencial, dejando en claro que ello sólo era posible con la reforma constitucional. Esta situación llevó a una política de presión frente a la UCR que por miedo a la judicialización y a una autorización de Menem por parte de una Corte Suprema de Justicia de la Nación que le era adscrita a producir lo que se conoció como el "Pacto de Olivos" en 1993 que abrió las puertas a la reforma constitucional de 1994 y por ende la posibilidad de reelección de Menem en 1995.

Las elecciones de 1995 muestran el primer gran cambio en nuestro sistema de partidos, ahí se ve claramente como el radicalismo es desalojado de su posición histórica a un tercer lugar y la irrupción del FG que alcanza a obtener el $30 \%$ de los votos convirtiéndose no solo en la gran sorpresa sino en la nueva alternativa.

\section{Tabla 3.}

Elección presidencial de 1995

\begin{tabular}{llcc}
\hline Fórmula & Partido & Votos & Porcentaje \\
\hline Carlos Menem - & Partido Justicialista - & & \\
Carlos Ruckauf & UCeDé & 8687319 & 49,94 \\
$\begin{array}{l}\text { José Octavio } \\
\text { Bordón - Carlos }\end{array}$ & $\begin{array}{l}\text { Frente País Solidario } \\
\text { Álvarez }\end{array}$ & & \\
$\begin{array}{l}\text { Poracio Mass- } \\
\text { accesi - Antonio }\end{array}$ & PSP-PSD & 5095929 & 29,3 \\
María Hernández & Unión Cívica Radical & 2956087 & 16,99
\end{tabular}




\begin{tabular}{llcc} 
Aldo Rico - Julio & $\begin{array}{l}\text { Movimiento por la } \\
\text { Fignidad y la Inde- }\end{array}$ & \\
Fernández Pez- & $\begin{array}{l}\text { Digndencia } \\
\text { zano }\end{array}$ & 294467 & 1,69 \\
Votos positivos & & 17394851 & 95,56 \\
Votos en blanco & 653434 & 3,59 \\
Votos anulados & & 125105 & 0,69 \\
\hline
\end{tabular}

Fuente: Dirección Nacional Electoral Argentina DINE Elaborado por: Autor.

Paradójicamente este nuevo escenario fue el inicio de la muerte del FG como alternativa y la vuelta a un bipartidismo en las elecciones presidenciales de 1999. Paralelo a ello, hay que aclarar que el espíritu hegemónico del menemismo tiene que ver en ello, ya que desde 1995 el menemismo comenzó a pensar en una nueva reforma constitucional o en una habilitación a la re reelección vía la Corte suprema de Justicia adscrita, este intento motivó que por cuestiones naturales, los líderes del FG y la UCR buscarán políticas comunes para frenar el intento de perpetuación en el poder, siendo las elecciones legislativas de 1997 la elección bisagra.

Ya en este punto podemos decir que el PJ se había transformado siguiendo la definición de Sartori (1999) en un partido predominante, debido a que por sí solo tenía garantizadas mayorías parlamentarias en diputados y senadores. Así es que para esas elecciones nace la coalición formada por ambos partidos denominada como Alianza que resultará ganadora en las elecciones legislativas. Finalmente Duhalde y el peronismo de la poderosa Provincia de Buenos Aires dejarían a Menem sin ningún tipo de chances convirtiendo a Duhalde en el candidato "natural" (el peronismo, salvo por primera vez en su historia en 1988, jamás seleccionó un candidato a presidente por elecciones internas o primarias) del Partido Justicialista (PJ). 
Las elecciones de 1999 volvieron a mostrar nuevamente un bipartidismo moderado, no ya entre el PJ y la UCR, sino entre el primero y la Alianza donde la UCR había ganado las elecciones internas donde Fernando de la Rúa había derrotado a Graciela Fernández Meijide en una elección interna entre afiliados de la UCR el FG e independientes que no fueron las primeras -la izquierda en los años 1980 había vivido el primer proceso-, este se constituyó en el más importante en virtud de los partidos que competían y el número de participantes en la misma. En esta elección, como en el 1983, con el Partido Intransigente (PI), en el 1989 la Unión de Centro Democrático (UCEDE), significó la aparición del partido Acción por la República (AR), del ex ministro Cavallo que obtuvo como tercer partido una importante representación parlamentaria.

La elección de 1999 significó no sólo el retorno el bipartidismo moderado sino también la segunda derrota del peronismo, pero ahora por primera vez compartiendo dos liderazgos como el de Menem y Duhalde.

\section{Tabla 4.}

Elección presidencial de 1999

\begin{tabular}{|c|c|c|c|}
\hline Fórmula & Partido & Votos & Porcentaje \\
\hline $\begin{array}{l}\text { Fernando de la Rúa - } \\
\text { Carlos Álvarez }\end{array}$ & $\begin{array}{l}\text { Alianza para el Trabajo, } \\
\text { la Justicia y la Edu- } \\
\text { cación UCR-FREPASO }\end{array}$ & 9167261 & 48,37 \\
\hline $\begin{array}{l}\text { Eduardo Duhalde - } \\
\text { Palito Ortega }\end{array}$ & $\begin{array}{l}\text { Concertación Justicial- } \\
\text { ista para el Cambio PJ- } \\
\text { UCeDé }\end{array}$ & 7253909 & 38,27 \\
\hline $\begin{array}{l}\text { Domingo Caval- } \\
\text { lo - Armando Caro } \\
\text { Figueroa }\end{array}$ & Acción por la República & 1937556 & 10,22 \\
\hline & 92 & & \\
\hline
\end{tabular}


Patricia Walsh - Ro- Alianza Izquierda Unida gelio De Leonardi PCA-MST $151276 \quad 0,8$

Votos postivos $18953063 \quad 95,49$

Votos en blanco 7018878

Votos anulados

Fuente: Dirección Nacional Electoral Argentina DINE Elaborado por: Autor.

El inicio del gobierno de la Alianza apareció signado por contradicciones que mostraron que las alianzas electorales sirven desde el punto de vista de lo electoral pero en los sistemas presidenciales no necesariamente sirven o son necesarias para la gobernabilidad. A los problemas que ya se arrastraban del modelo económico neoliberal -el cual de la Rúa jamás intentó modificar-, se sumaron los problemas típicos de la falta de liderazgo y gobernabilidad, a la renuncia del Vice Presidente Carlos "Chacho" Álvarez se sumaron denuncias de corrupción. La caída del gobierno de la Alianza entre el 19 y el 20 de Diciembre de 2001 significó además de la profundidad de la crisis de legitimidad el fin del sistema de partidos tradicional que había tenido la Argentina. Tomando como referencia los conceptos de Ware (1996) y haciendo hincapié en los factores sociológicos, podemos decir que los cambios sociales profundos a diferencia de los cambios menos drásticos afectan de algún modo el sistema de partidos.

Si bien la crisis exterminó el gobierno, implicó elementos más profundos que perduran hasta nuestros días, como por ejemplo:

$1^{\circ}$ ) Crisis de la UCR como partido histórico nacional, a partir de allí el radicalismo jamás pudo encarnar ni como partido nacional, ni con un dirigente del seno partidario un proyecto triunfante como alternativa de poder real. 
$\left.2^{\circ}\right)$ La connotación negativa de la palabra "alianza", como si las coaliciones o asociaciones entre partidos fueran la reencarnación del mal en la tierra.

$3^{\circ}$ ) Aunque en menor medida que en el radicalismo, la crisis también se vio en el peronismo no como movimiento político sino en su herramienta política que es el PJ.

El gobierno de concertación parlamentaria de Duhalde (2002-2003) llega a su fin con la muerte de los manifestantes Kosteki y Santillán, lo que aceleró la salida del gobierno y el llamado a elecciones para elegir a un presidente.

El contexto de crisis sistémica es ambiente propicio para el desmantelamiento de los sistemas de partidos tradicionales, lo marca la experiencia histórica pasada y reciente, el mani pulite y la tangentopoli italiano de los años 1990 hizo lo propio con el modelo del pentapartito Italiano; la crisis venezolana de esa misma década tuvo las mismas consecuencias con los partidos que sostenían la gobernabilidad desde el pacto del punto fijo en 1958; sin ir más lejos, los actuales problemas que vive España son producto de la crisis sistémica y el fin del viejo modelo partidario vigente desde la transición española de la década del 1980.

Italia y Venezuela en la década del 1990, Argentina en 2001 y España actual vivieron y viven los problemas que supone para una democracia la caída de su viejo y tradicional sistema de partidos, producto de una crisis de legitimidad que afecta prácticamente la totalidad de los estamentos del Estado. No cabe dudas que los gobiernos democráticos más sólidos son los que poseen sistemas bipartidistas concentrados como sucede en EE.UU o moderados como era el caso de Argentina o España. Bajo estos sistemas no solo los gobiernos son más fuertes, sino que también el establecimiento de los consensos se torna mucho más previsible pero fundamentalmente institucional. 
Siguiendo con el caso argentino que es el que nos ocupa en este artículo, el fin del bipartidismo no significó la muerte de las estructuras tradicionales (PJ-UCR) sino una nueva modalidad que significó la reconstitución de sus dirigencias y sus doctrinas por fuera de las estructuras partidarias sin que estas terminen de desaparecer. Estamos en presencia del giro más importante de nuestro sistema de partidos, que es la salida del bipartidismo y el ingreso a una fragmentación absoluta en las elecciones de 2003 al presentarse, siempre siguiendo las categorías de Sartori un multipartidismo segmentado.

En base a esta realidad, Scott Mainwaring (1993) anticipa el problema que supone para el sistema presidencial el multipartidismo, el cual exacerba los enfrentamientos entre ejecutivo y legislativo. En sintonía, Sartori (2003) también considera el bipartidismo como el sistema clásico de los regímenes presidenciales y el aporte que producen para la gobernabilidad, esta idea la refuerza con la necesidad de reformas electorales que introduzcan sistemas electorales mayoritarios a fin de salir del multipartidismo y recomponer un nuevo sistema bipartidista.

En consonancia, Duverger (1974) también considera que los sistemas multipartidistas, en primer lugar, conducen al poder a coaliciones desarticuladas que deben realizar todo tipo de pactos para lograr la gobernabilidad o para ser competitivas; en segundo lugar, la heterogeneidad y fragmentación hacen imprevisible la direccionalidad del gobierno y pone en jaque la estabilidad del sistema; y en tercer lugar, fomenta la radicalización de las posturas y resta responsabilidad a los dirigentes.

En relación a lo expresado, Nohlen (1998) no solo determina la importancia de las elecciones competitivas garantizadas por los pilares básicos del sistema liberal como son el voto secreto, universal, igualitario y libre; sino también por tres factores estructurales que la determinan como son: 
$\left.1^{\circ}\right)$ La estructura del sistema social que habla de las características económico sociales existentes.

$2^{\circ}$ ) La estructura del sistema político, ya sea parlamentario o presidencial, unitario o federal.

$3^{\circ}$ ) La estructura del sistema de partidos dado por el tamaño, número y distancia ideológica entre ellos.

Ahora, plantea Nohlen que en sociedades menos homogéneas con sistemas multipartidistas, las elecciones no cumplen funciones que sí se producen cuando se dan bajo sistemas bipartidistas, como es en primer lugar la no resolución de cuestiones ligadas al ejercicio del poder, las cuales deben ser resueltas posteriormente en negociaciones post electorales; en segundo lugar, canalización de conflictos políticos mediante procedimientos pacíficos.

Centrándonos en el caso particular de la Argentina que nos ocupa en este artículo, las elecciones presidenciales del año 2003 marcaron una atomización muy importante al punto que el candidato más votado fue Carlos Menem que no llegó a reunir el $25 \%$ de los votos válidamente emitidos, lo que llevó a la conformación de un sistema de partido multipartidista.

Esa elección fue la consecuencia de la ruptura de los partidos históricamente tradicionales en Argentina. En primer lugar, el peronismo en un congreso partidario renunció por primera vez al monopolio que históricamente tuvo del peronismo mediante el Partido Justicialista como herramienta electoral y habilitó como una forma de evitar internas o primarias para la sección de candidatos la conformación de diferentes frentes encabezados por dirigentes del peronismo, así aparecen las candidaturas de Menem, Kirchner y Rodríguez Saá representando todos ellos al peronismo o las diferentes vertientes de este. En segundo lugar, el radicalismo como 
estructura partidaria vivió un largo y controvertido proceso de elecciones internas fiel a su tradición entre Moreau y Terragno que terminó con denuncias cruzadas por fraude electoral que termina finalmente con la candidatura oficial de Moreau por el partido; pero a pesar de ello, no oficialmente vivió un proceso similar al peronismo, ya que dirigentes de la UCR rompen con el partido y presentan candidaturas independientes por fuera del partido como son los casos de Elisa Carrió y Ricardo López Murphy.

Esta coyuntura marcó el fin de los partidos políticos como tradicionalmente se los había conocido y marca el inicio de un proceso de desgaste y descomposición del sistema de partidos que hasta el día de hoy no se ha podido reconfigurar. Sin embargo, queda demostrado el multipartidismo en la distribución de los votos en dicha elección como demuestra la tabla 5.

\section{Tabla 5.}

Elección presidencial de 2003

\begin{tabular}{llcc}
\hline Fórmula & Partido & Votos & Porcentaje \\
\hline $\begin{array}{l}\text { Carlos Menem - } \\
\text { Juan Carlos Rome- } \\
\text { ro }\end{array}$ & $\begin{array}{l}\text { Frente por la Leal- } \\
\text { tad-UCeDé }\end{array}$ & 4740907 & 24,45 \\
$\begin{array}{l}\text { Néstor Kirchner - } \\
\text { Daniel Scioli }\end{array}$ & Frente para la Victoria & 4312517 & 22,24 \\
$\begin{array}{l}\text { Ricardo López } \\
\text { Murphy - Ricardo }\end{array}$ & & & \\
$\begin{array}{l}\text { Gómez Diez } \\
\text { Adolfo Rodríguez } \\
\text { Saá - Melchor }\end{array}$ & Recrear & 3173475 & 16,37 \\
Posse & Libertad Pop. Unión y & & \\
& & 2735829 & 14,11
\end{tabular}


Elisa Carrió - Gus-

tavo Gutiérrez

ARI

2723574

14,05

Leopoldo Moreau -

Mario Losada

UCR

453360

2,34

Votos positivos

19387895

97,28

Votos en blanco

196574

0,99

Anulados

345642

1,73

Fuente: Dirección Nacional Electoral Argentina DINE

Elaborado por: Autor.

Como veremos en el próximo ítem, esta situación de multipartidismo comienza a revertirse, dando al sistema de partidos políticos argentino un nuevo giro y una nueva transformación. Al igual que lo ocurrido en muchos países que viven crisis sistémicas que erosionan el viejo esquema partidario como es el caso venezolano por ejemplo, se da un fenómeno muy particular que va de la mano de la demanda particular de la sociedad de tener presidencialismos fuertes, así es el caso de Néstor Kirchner quien asume la presidencia con el $22 \%$ de los votos, rápidamente va construyendo un poder cada vez más grande hasta darle a la Argentina un cambio en términos de una nueva concepción del poder. Hasta tal punto es la popularidad de lo que se conoce como el kirchnerismo que lleva el sistema de partidos a una situación de partido predominante más notoria que la que se había producido en los años 1990 con el menemismo. Así se inicia una nueva etapa de ruptura donde pasamos de un sistema multipartidista a otro de partido predominante, aunque el partido, a partir de la crisis del 2001 pasa a ser un elemento secundario del sistema, ya que se inicia desde 2003 una competencia entre nombres y figuras más que entre partidos, lo cual lesiona el ejercicio institucional de la democracia. 


\section{Contexto actual}

\section{La crisis partidaria y la irrupción de los personalismos}

Como se ha indicado anteriormente, desde el 2003 el kirchnerismo, con un discurso populista y rupturista en relación a la década del 1990 y heredero de una transición ordenada entre 2001 y 2003, lo cual suponía también que en ese período se pagaron los costos de la devaluación y salida del esquema de convertibilidad de los noventa, comenzó a disfrutar de un comercio internacional favorable y de una pronta recomposición del mercado laboral que lo llevó a transformarse en una fuerza política que iba a tener la supremacía electoral en toda la década.

Esta rápida mejora y la legitimidad de ejercicio en el poder hacen que el Frente para la Victoria (FPV), sello electoral con el que compitió Kirchner en 2003, concentre bajo su seno al PJ y desde allí se dé la división entre kirchnerismo y duhaldismo por la conducción del PJ, disputa que quedará para el lado de Kirchner cuando en la elección de 2005 por la senaduría de la provincia de Buenos Aires, Kirchner y Duhalde juegan a sus esposas al frente de las boletas electorales siendo Cristina Fernández de Kirchner la amplia ganadora en lo que se conoció como la madre de todas las batallas. A partir de esta elección, la estructura originaria del PJ provincial donde se incluían intendentes y viejos líderes territoriales que apoyaron a Duhalde en esa elección, se alinearon automáticamente al FPV y desde ahí se inicia con tendencia hegemónica, sin llegar a serlo pero de situación predominante en el sistema de partidos políticos.

En todo el periodo 2003-2015 se va a dar sistemáticamente una misma situación, salvo el FPV, por la sencilla razón de que se construyó y organizó desde el poder por un sistema verticalista que tenía al Estado y sus recursos como premios y castigos; el resto de las fuerzas políticas compite en las diferentes elecciones con distintos nombres productos de múltiples 
alianzas que se fueron produciendo circunstancialmente como una suerte de estrategia del "sálvese quien pueda" para enfrentar una derrota segura frente al kirchnerismo. La desorientación de la oposición contribuyó en gran medida, producto de su falta de competitividad, pero también de poder articular acuerdos programáticos serios a la despartidización de nuestra democracia.

La construcción desde el Estado por parte del kirchnerismo quedó demostrada en la elección presidencial de 2007 donde se presenta la concertación que significó aglutinar no solo al peronismo sino también a gobernadores e intendentes provenientes desde la UCR que pasaron a formar parte del FPV, la síntesis de ello fue la inclusión de Julio Cobos como candidato a vicepresidente. Obviamente ese acuerdo no fue ideológico sino que tiene que ser visto dentro del sistema de premios y castigos que se hacía desde el poder y fundamentalmente del envío discrecional de fondos para obras públicas por parte del Poder Ejecutivo.

\section{Tabla 6.}

Elección presidencial 2007

\begin{tabular}{llcc}
\hline Fórmula & Partido & Votos & Porcentaje \\
\hline $\begin{array}{l}\text { Cristina Fernández - } \\
\text { Julio Cobos }\end{array}$ & Frente para la Victoria & 8651066 & 45,29 \\
$\begin{array}{l}\text { Elisa Carrió - Rubén } \\
\text { Giustiniani }\end{array}$ & Coalición Cívica & 4401981 & 23,04 \\
$\begin{array}{l}\text { Roberto Lavagna - } \\
\text { Gerardo Morales }\end{array}$ & Una Nación Avanzada & 3229648 & 16,91 \\
$\begin{array}{l}\text { Alberto Rodríguez } \\
\text { Saá - Héctor María } \\
\text { Maya }\end{array}$ & $\begin{array}{l}\text { Frente Justicia, Unión } \\
\text { y Libertad }\end{array}$ & 1458955 & 7,64
\end{tabular}


Fernando Solanas -

Ángel Cadelli

Ricardo López

Murphy - Esteban

Bullrich

Votos positivos

Votos en blanco

Votos nulos
Partido Socialista

Auténtico

301265

1,58

Recrear

273015

1,43

$19102211 \quad 92,4$

$1330885 \quad 6,44$

$240074 \quad 1,16$

Fuente: Dirección Nacional Electoral Argentina DINE Elaborado por: Autor.

Esa desorientación de la oposición, aportó además un elemento de atomización característico del sistema multipartidista pero en un contexto de un partido con tendencia predominante como era el kirchnerismo, razón por la cual ensanchó aún más el peso del oficialismo y le quitó a la oposición peso, en tanto a su posibilidad cierta de disputar el poder. Podemos decir que el sistema de partidos en Argentina se caracterizó durante toda esta etapa por los siguientes elementos:

A. El kirchnerismo (FPV) como partido predominante producto de estas características:

i. Utilización del poder del ejecutivo para desarrollar política de alianzas mediante premios y castigos a la hora de distribuir fondos u obras públicas en un sistema hiperpresidencial donde los controles son ineficaces en el mejor de los casos.

ii. El esquema anterior le permitió derrotar al Duhaldismo y concentrar el poder hegemónico dentro del peronismo.

iii. Del mismo modo, esa lógica hizo que tentara a dirigentes 
que tenían cargos por partidos opositores para que pasen a formar parte del FPV, lo cual le permitió quebrar el poder territorial de la oposición.

iv. Control de prácticamente todos los territorios electorales importantes, ya sea mediante gobiernos propios o aliados.

v. La versatilidad ideológica y el carácter movimientista del peronismo permiten su rápida reconfiguración más allá del contexto histórico.

B. La oposición por su parte, ya sea por acción u omisión tampoco reaccionó frente a este poder, sino que por el contrario contribuyó a hacerlo cada vez más concentrado producto de estos elementos:

i. Incapacidad de institucionalizar un partido o alianza electoral medianamente estable que la identifique como alternativa. Lejos de ello, implosionó sistemáticamente en cada elección cualquier tipo de estabilidad de su fuerza.

ii. El radicalismo principalmente como partido histórico y con peso en el interior no tuvo capacidad para retener dirigentes los cuales fueron incorporados al FPV. Este elemento fue característico de todas las fuerzas opositoras.

iii. Ineficacia a la hora de constituir planes y programas alternativos que permitieran discusiones horizontales, programáticas y no maniqueas con el oficialismo, donde lejos de contribuir a un debate serio y propositivo, la oposición entró en la lógica binaria, ingresando a una estrategia donde el FPV se sentía cómodo y sacaba rédito de ello.

iv. Mostró muchas veces sectarismo, protagonismo extremo 102 
y presentó otros liderazgos de carácter mesiánicos que contribuyeron a fortalecer en vez de debilitar al oficialismo.

v. Incapacidad de proyectar a un candidato ganador en elecciones de medio término en elecciones ejecutivas. Tal es el caso de Francisco de Narváez que derrota a Kirchner en las elecciones legislativas de 2009 pero sin lograr consolidarse en una elección de cargo ejecutivo.

vi. Finalmente, fue incapaz de aprovechar la Ley de Primarias Abiertas Simultáneas y Obligatorias para competir y seleccionar en un proceso democrático un candidato que la unificara. Esto llevó a que en esa elección se produjera el récord de diferencia entre el partido ganador y el segundo en toda la historia argentina que fue de 37,19\% de diferencia a favor de Cristina Kirchner.

\section{Tabla 7.}

Elección presidencial 2011

\begin{tabular}{|c|c|c|c|}
\hline Fórmula & Partido & Votos & Porcentaje \\
\hline $\begin{array}{l}\text { Cristina Fernández - } \\
\text { Amado Boudou }\end{array}$ & Frente para la Victoria & 11865055 & 54,11 \\
\hline $\begin{array}{l}\text { Hermes Binner - Norma } \\
\text { Morandini }\end{array}$ & $\begin{array}{l}\text { Frente Amplio Progre- } \\
\text { sista }\end{array}$ & 3684970 & 16,81 \\
\hline $\begin{array}{l}\text { Ricardo Alfonsín - Javi- } \\
\text { er González Fraga }\end{array}$ & $\begin{array}{l}\text { Unión para el Desar- } \\
\text { rollo Social }\end{array}$ & 2443016 & 11,14 \\
\hline $\begin{array}{l}\text { Alberto Rodríguez Saá - } \\
\text { José María Vernet }\end{array}$ & Compromiso Federal & 1745354 & 7,96 \\
\hline
\end{tabular}


Eduardo Duhalde - Ma-

\begin{tabular}{llcc} 
rio Das Neves & Frente Popular & 1285830 & 5,86 \\
$\begin{array}{l}\text { Jorge Altamira - Chris- } \\
\text { tian Castillo }\end{array}$ & $\begin{array}{l}\text { Frente de Izquierda y } \\
\text { de los Trabajadores }\end{array}$ & 503372 & 2,3 \\
$\begin{array}{llc}\text { Elisa Carrió - Adrián } \\
\text { Pérez }\end{array}$ & Coalición Cívica ARI & 399685 & 1,82 \\
Votos positivos & & 21927282 & 95,52 \\
Votos en blanco & 803362 & 3,5 \\
Votos nulos & & 225741 & 0,98 \\
\hline
\end{tabular}

Fuente: Dirección Nacional Electoral Argentina DINE Elaborado por: Autor.

Como todos sabemos, la muerte de Néstor Kirchner en 2010 supuso que la sucesión presidencial viviera lo que en los presidencialismos se conoce como el síndrome del "pato rengo". En este caso el impedimento constitucional de reelección indefinida llevó al kirchnerismo a la misma lógica que había vivido el menemismo 15 años atrás a buscar una reforma que eternizara el proyecto en el poder.

Más que por la lógica del destino, sino por la propia lógica del peronismo, Cristina Fernández chocó al igual que Menem con la misma piedra, es decir el peronismo, como había sido Duhalde al final de la década del noventa, o Kirchner con Duhalde en 2005: otro peronista fue el encargado de derrotar esta vez al oficialismo desde un nuevo partido conformado en la figura de su líder Sergio Massa, que con el Frente Renovador (FR) debuta en las elecciones de 2013 y derrota al FPV en la Provincia de Buenos Aires, reeditando lo que se conoció nuevamente como la madre de todas las batallas.

Esta elección abrió la puerta a una nueva reconfiguración del sistema de partidos en la elección de 2015 donde volvemos a ver el surgimiento de un sistema altamente competitivo, que permitió la derrota del oficialismo en 
los principales centros urbanos y a nivel nacional, habilitando la alternancia y pasando a un esquema de distribución del poder más equilibrado. En esa elección nos encontramos con un bipartidismo extremadamente moderado donde aparece una tercera fuerza (Unidos por una Nueva Alternativa) que supera el $20 \%$ de los votos y se transforma en árbitro del consenso legislativo.

\section{Tabla 8.}

Elección presidencial 2015

\begin{tabular}{llcc}
\hline Fórmula & Partido & Votos & Porcentaje \\
\hline $\begin{array}{l}\text { Daniel Scioli - } \\
\text { Carlos Zannini }\end{array}$ & Frente para la Victoria & 9338449 & 37,08 \\
$\begin{array}{l}\text { Mauricio Macri - } \\
\text { Gabriela Michetti }\end{array}$ & Cambiemos & 8601063 & 34,15 \\
$\begin{array}{l}\text { Sergio Massa - } \\
\text { Gustavo Sáenz }\end{array}$ & Unidos por una Nueva & & \\
Nicolás del Caño & & 5386965 & 21,39 \\
- Myriam Breg- & Frente de Izquierda y & & \\
man & de los Trabajadores & 812530 & 3,23 \\
$\begin{array}{l}\text { Margarita Stol- } \\
\text { bizer - Miguel }\end{array}$ & & \\
$\begin{array}{l}\text { Olaviaga } \\
\text { Adolfo Rodríguez }\end{array}$ & Progresistas & 632551 & 2,51 \\
$\begin{array}{l}\text { Saá - Liliana Ne- } \\
\text { gre de Alonso }\end{array}$ & Compromiso Federal & 412557 & \\
Votos positivos & & 25184135 & 96,68 \\
Votos blancos & & 664739 & 2,55 \\
Votos en nulo & & 199446 & 0,77 \\
\hline
\end{tabular}

Fuente: Dirección Nacional Electoral Argentina DINE Elaborado por: Autor. 
La disyuntiva es hasta qué punto la alianza gobernante (Cambiemos) se puede mantener unida y hasta qué punto el kirchnerismo pueda superar las dificultades que está mostrando para mantener la homogeneidad del FPV, que está viviendo el proceso de descomposición que caracterizó a la oposición argentina durante su etapa de gobierno, demostrando la poca capacidad de aglutinamiento y contención que tienen los partidos desde la oposición para sostener su estructura.

Más allá de esto, lo que caracterizó la oferta electoral fueron los nombres particulares de líderes o personajes que construyeron tras su figura alternativas electorales independientemente del sello partidario, lo cual lesiona la democracia partidaria típica de los países donde los partidos son fuertes y seleccionan mediante procesos internos sus propias élites dirigenciales. Esta coyuntura marca un retroceso en la democracia argentina, porque lejos de ampliarse los canales de participación y decisión, llegamos a una instancia donde las oligarquías partidarias toman el control y desplazan a los afiliados y simpatizantes de las resoluciones más trascendentales de los partidos.

Este es el desafío que supone la recomposición del sistema de partidos, única alternativa para salir de los personalismos e institucionalizar métodos y políticas necesarias para los consensos democráticos pero desde los partidos y no desde los dirigentes. Los principales acuerdos y votaciones en el congreso de la nación fueron conocidos por la sociedad en anuncios públicos por los dirigentes de todos los partidos políticos, corriendo la participación al mero espectáculo de observar por TV la decisión de los dirigentes.

Finalmente, las plataformas electorales son realizadas por técnicos o especialistas que basan la consistencia de las mismas por encuestas de opinión pública, lo cual para satisfacer las demandas sociales, muchas veces contradicen los postulados históricos de los partidos, lo cual afecta también la credibilidad del mismo en el corto plazo. El funcionamiento pleno de los partidos supone que las políticas de fondo deben ser discutidas desde un plano 
institucional con la mayor participación posible de afiliados y simpatizantes.

Esta característica asumida por el sistema de partidos torna conflictivo el sistema democrático, los personalismos y la falta de estructuras partidarias que discutan y diriman en procesos internos institucionalizados claros es necesaria como cuestión sine qua non para la democracia. Reestructurar un sistema de partidos ordenado es la primera cuestión que debe analizarse y la cuenta pendiente luego de tres décadas del inicio de la transición democrática. Este es el verdadero desafío para el sistema, reconstruir esos espacios de participación y decisión para lograr una mejor representatividad política. 


\section{Bibliografía}

Duverger, M. (1974). Constitutions et documents politiques. París: Presses Universitaires de France.

Mainwaring, S. (1993). "Presidentialism, Multipartism, and Democracy: The Difficult Combination". En Comparative political studies, 26(2), 198-228.

Nohlen, D. (1998). Sistemas electorales y partidos políticos (Vol. 2). México: Fondo de Cultura Económica.

Sartori, G. (1999). Partidos y sistemas de partidos: Marco para un análisis. Madrid: Alianza.

Sartori, G. (2003). Ingeniería constitucional comparada: una investigación de estructuras, incentivos y resultados. México: Fondo de Cultura Económica.

Ware, A. (1996). Political parties and party systems. Oxford: Oxford University Press. 